\title{
Testing of an automatic earthquake detection method on data from Station Nord, Greenland
}

\author{
Nasim Karamzadeh, Peter H. Voss and Gholam D. Javan
}

Earthquakes are continuously monitored by a global network of several thousand seismic stations equipped with highly sensitive digital seismometers. The Geological Survey of Denmark and Greenland (GEUS) takes part in it by operating five seismic stations in Denmark and 18 in Greenland, some of the latter in collaboration with international partners. There are two main ways of detecting earthquakes from digital recordings of seismometers: (1) by a manual review of the data by an expert in processing seismic earthquake signals and (2) by an automatic method that uses a computerised algorithm to analyse the recordings.

Since the beginning of earthquake recording, earthquake detection at GEUS has been based on the manual review method. There are several reasons why an automatic detection procedure has not yet been implemented at GEUS: (1) historically, the staff at GEUS have conducted high-quality manual detection of earthquakes, based on a long tradition of manual seismogram analysis (Lehmann 1954), (2) the ambient noise level in Denmark is generally too high for small local earthquakes to be detected automatically and (3) in Greenland, the distance between the seismometers is too long for automatic methods. Previous tests on GEUS data showed that automatic detection using the so-called standard method resulted in a very high number of false detections, and the effort needed to distinguish real earthquake signals from noise signals was much greater than that needed in the manual method. In addition, the automatic method detected fewer earthquakes than the manual analysis. Therefore, new automatic methods are needed to extract real earthquake signals from the background noise.

In this article, we present results from testing a newly developed automatic detection method based on analysis of the frequency content of seismic signals. The aim of the study was to investigate whether the automated method can be used in Greenland or whether the manual procedure is still superior. The new method was tested on seismic data from Station Nord, which was selected because it is located in a region with many earthquakes, and because there are no nearby seismograph stations to support the measurements. The closest station is located at Danmarkshavn c. $540 \mathrm{~km}$ to the south. Therefore, the majority of earthquakes that occur in this region are only registered at Station Nord.

\section{Earthquakes in the Station Nord region}

Station Nord is located in eastern North Greenland, in a region where a major tectonic factor is the spreading that occurs along the rift zones in the northern North Atlantic and the Arctic Ocean (Døssing et al. 2010), and which gives rise to high seismic activity (Fig. 1). Another tectonic factor is postglacial isostatic rebound that was the source of three major earthquakes in 1971, 1987 and 1993 (Chung 2002), with magnitudes of 5.1, 5.5 and 5.4 on the Richter scale. Apart from the seismicity observed at the rift zone, most of the earthquakes in the region occur to the south and west of Station Nord (Fig. 1; Gregersen 1982). The earthquake hazard in the region is low, but represents the highest in Greenland (Voss et al. 2007). The hazard was illustrated by a strong tremor felt at Station Nord on 30 August 2005, caused by a magnitude 4.2 earthquake with an epicentre only $20 \mathrm{~km}$ away.

\section{Automatic earthquake detection}

The standard automatic earthquake detection method is known as the Short Term Average versus Long Term Average (STA/LTA) method (Havskov \& Alguacil 2010). The basis of this method is two running time windows that both compute the average amplitude of the signal, one with a short

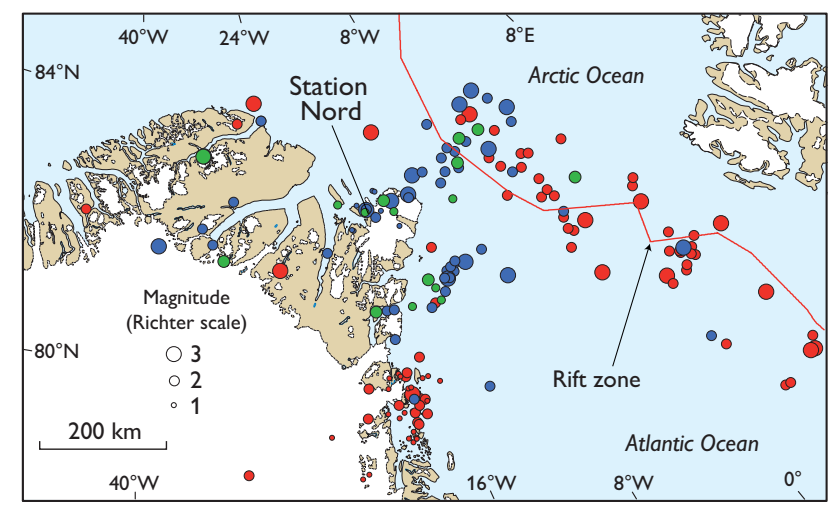

Fig. 1. Map of the region between north-eastern Greenland and Svalbard. Red dots: Epicentres located by GEUS using manual detection methods. Blue dots: Epicentres located using automatic detection method. Green dots: Located by both methods. 

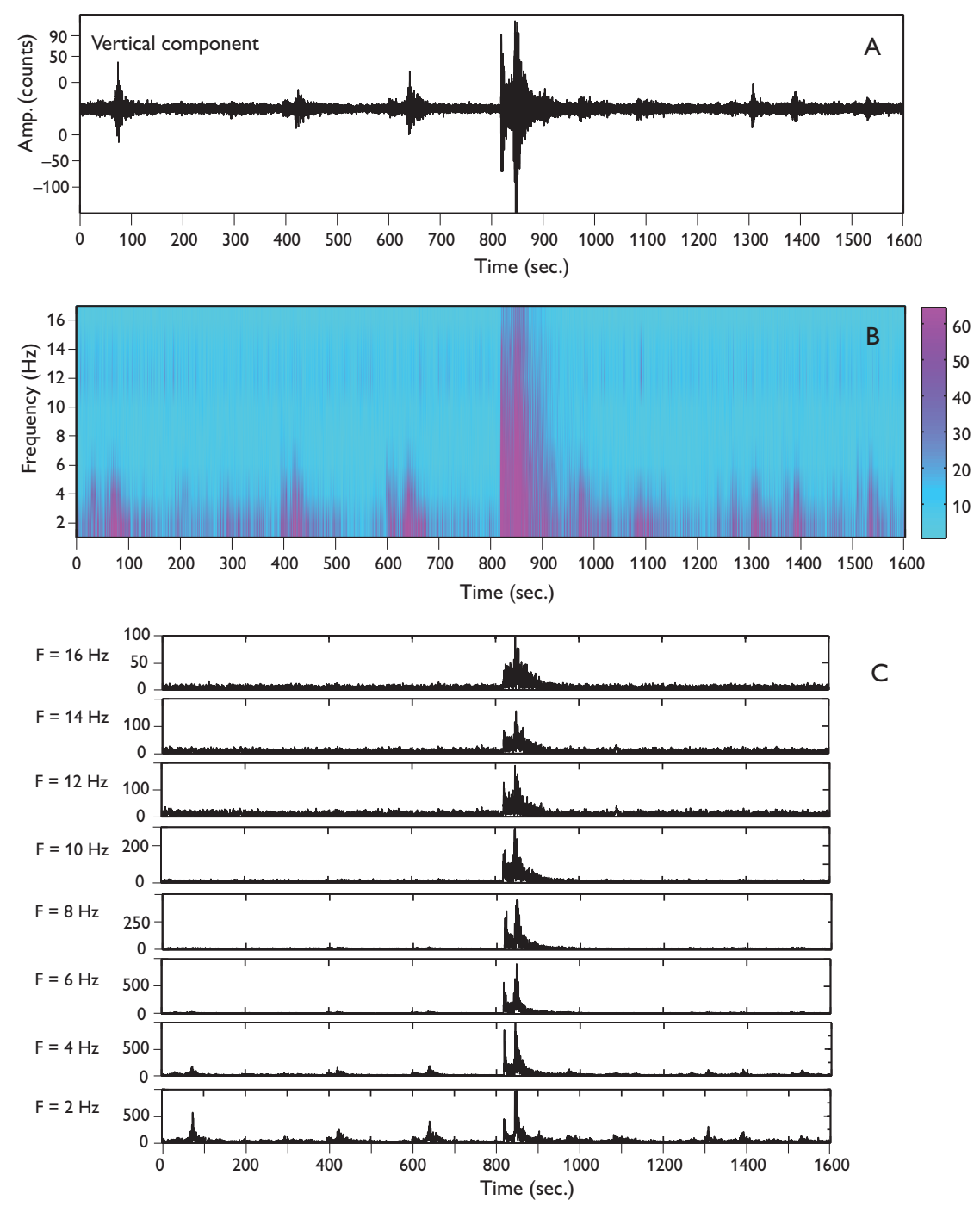

Fig. 2. A: Vertical component of a continuous seismogram, which included a signal from an earthquake and several short-duration, high-energy perturbations. B: Normalised spectrogram produced by short-time Fourier transformation for frequencies of $2-16 \mathrm{~Hz}$ with $2 \mathrm{~Hz}$ steps. The colours (scaled to use full colour spectrum) represent an estimate of the energy contained at the given frequency, within a short window (about $0.6 \mathrm{~s}$ ). At $800-900 \mathrm{~s}$, when an earthquake signal appears in the seismogram, the colours on the spectrogram change, which indicates an order of magnitude increase in the seismic energy over all frequencies. The detection of changes in energy over a proper range of frequencies, corresponding to the frequency content of the earthquake signals, led to the detection of an earthquake. Other increases in the energy level are seen at several other times, for example at 600-700 s. These peaks are only seen at low frequencies. C: Another representation of the same spectrogram showing the variation of local spectral energy for each frequency band over time. A sharp increase is seen at the onset of the earthquake signal, whereas the perturbation of energy in the background noise has disappeared at higher frequency bands. duration (STA) and the other with a long duration (LTA). The LTA represents the stable background noise level of the seismic signal, whereas the STA will increase rapidly at the onset of a seismic signal. The signal is often band pass filtered to lower the ambient noise. The earthquake is detected when the STA/LTA exceeds a predefined threshold level (e.g. 10). The basic parameters required for this method are (1) the band pass filter, (2) the duration of STA and LTA and (3) the STA/LTA threshold level. The disadvantage of this method is that it triggers with all ground shaking that exceeds the threshold level. So, if the STA/LTA threshold level is set low to detect small earthquakes, many detections that are nothing but noise or bogus events lead to much additional manual post-processing. A high number of noise detections can be avoided by setting the STA/LTA at a higher threshold level, but then important earthquakes may not be detected.

In many seismic networks, automatic detection is operated with a low STA/LTA threshold level, but to avoid a lot of manual post-processing of detected signals, an additional criterion is applied before they are forwarded to manual processing. It states that the detection is only valid if the signal is also detected by a number of other seismic stations (e.g. 3) within a predefined time window; otherwise the detection is disregarded as being seismic noise. However, in order to use this criterion in the detection of small earthquakes, the network must include a large number of seismic stations located less than $100 \mathrm{~km}$ from each other. This is not the case in Greenland where the distance between the stations is around $300 \mathrm{~km}$ in western Greenland and around $600 \mathrm{~km}$ in eastern Greenland. Alternative methods are therefore required, and we have chosen an automatic method based on analysis of the short-time Fourier transform of the data. In addition to event detection, the automatic method also provides phase picks, magnitude measurements and azimuth estimates, but the primary aim of this study was to test the performance of the automatic event detections. 
A spectrogram produced by short-time Fourier transform is a very useful tool in seismology because it can provide an image indicating the time at which a burst of energy occurs on a seismogram, in addition to the spectral composition of the signal (Gibbons et al. 2008). The event detection algorithm used in this study inspects the temporal variation of the signal spectrogram calculated in frequency bands corresponding to the frequency content of local and regional earthquakes (e.g. $2-16 \mathrm{~Hz}$ ). For detected events, the $P$ - and $S$-phases are picked. An example of a recorded seismogram with an earthquake signal and corresponding spectrogram is shown in Fig. 2 where an earthquake is observed on a seismogram at an approximate time of $800 \mathrm{~s}$. Obvious changes in the colour of the spectrogram take place over a wide range of frequencies along the time axis, which indicate the arrivals of earthquake energy (Fig. 2B). Accordingly, the detection of a change in energy pattern over a pre-defined range of frequencies, corresponding to the frequency content of the earthquake signals, leads to the detection of an earthquake. The plots presented in Fig. 2C show the variation of energy for each frequency band, corresponding to the above spectrogram. These plots provide another representation of the spectrogram. The problem of detecting an earthquake on seismograms is now reduced to detecting sharp increases in the individual time series representing spectral energy versus time (the plot shown in Fig. 2C). To avoid false detections due to seismic noise with a frequency content overlapping the analysed frequencies, only detections made in most of the frequency bands are accepted. For instance, detections should be made at about the same time in at least five out of eight frequency sub-bands for a given spectrogram (Fig. 2B). Three missing detections are allowed, because this may happen for small events and noisy backgrounds, or low signal to noise ratio in some frequency bands. To reduce the false detection rate, all three components (vertical, N-S and E-W) of the seismograms are used in the event detection procedure.

\section{Results}

To test the automatic detection method, Station Nord data from 6 July 2010 to 6 March 2011 were used. Prior to this period, the digitising unit of the seismometer had been upgraded to sample at $100 \mathrm{~Hz}$. Earlier, the instrument had sampled at $20 \mathrm{~Hz}$; this limits earthquake analysis to frequencies below $10 \mathrm{~Hz}$, which the automatic method was not prepared for. Station Nord is equipped with a Streckeisen STS-2 sensor and a Quanterra Q330 digitiser. The automatic method analyses data from all three components of the sensor, using 24 hour data files. The data are band pass filtered between 0.95 and $20 \mathrm{~Hz}$ before the detection algorith $\mathrm{m}$ is applied. The
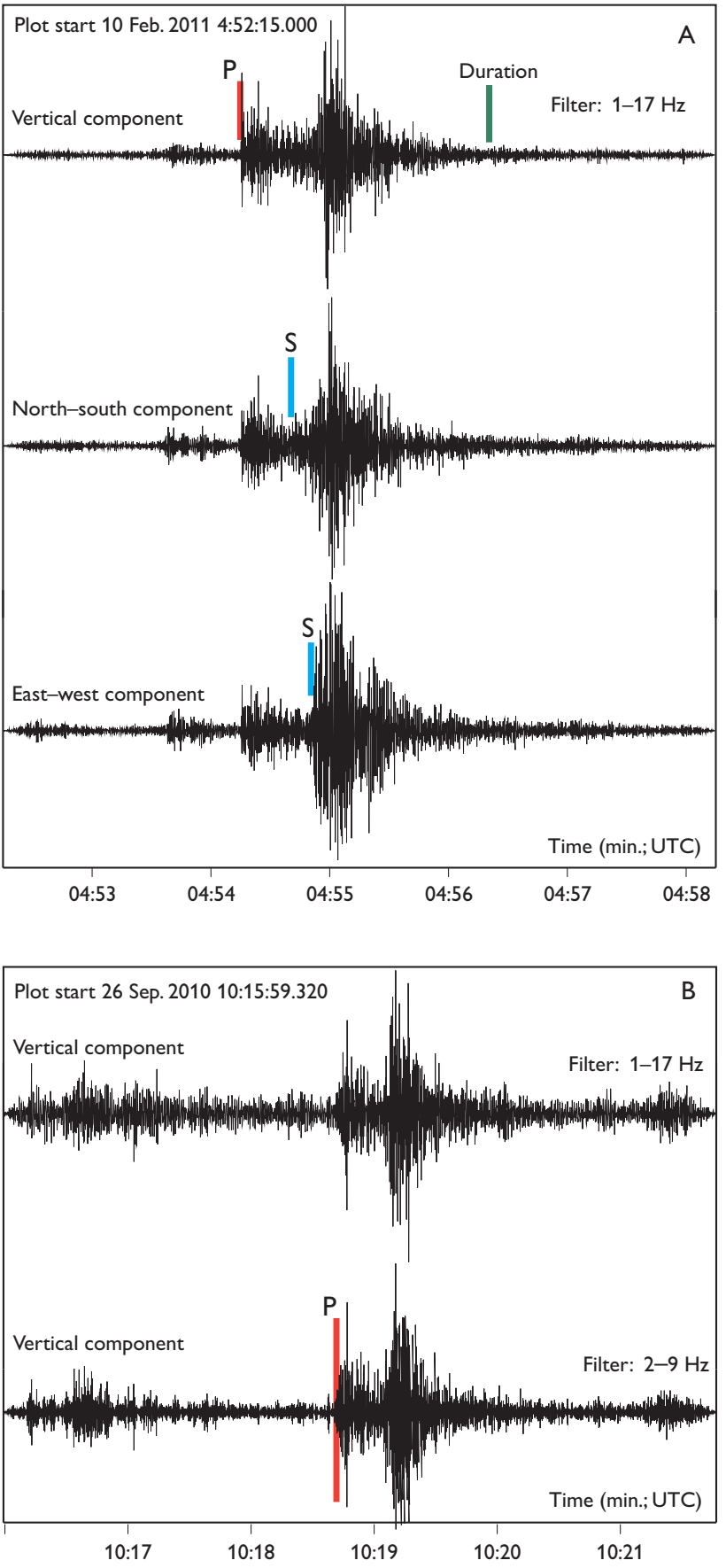

Fig. 3. A: Seismogram of a magnitude 2.2 earthquake filtered with a 1-17 $\mathrm{Hz}$ band pass filter containing the frequencies used for the automatic detection. The epicentre was located $354 \mathrm{~km}$ south-south-east of Station Nord. The automatic $P$-phase $(\mathbf{P})$ was kept in the review, but the automatic $S$-phase $(\mathbf{S})$ was repicked moving the epicentre $118 \mathrm{~km}$. Automatic $S$ pick is seen on the north-south channel, manual $S$ pick is seen on the east-west channel and the automatic tremor duration is seen on the vertical channel. B: Vertical component of an earthquake not detected by the automatic method. Top trace: data with the $1-17 \mathrm{~Hz}$ filter used by the automatic method. Bottom trace: data with the $2-9 \mathrm{~Hz}$ filter used by the manual method. The earthquake had a magnitude of 1.1 and was located $234 \mathrm{~km}$ east-south-east of Station Nord. UTC: Universal Time, Coordinated. 
detections are stored in the international SEISAN format. During the test period, 156 detections were recorded with the automatic method. A review of these detections showed that 13 were due to sensor calibrations or sensor noise, and 52 were caused by other noise signals such as man-made noise. The remaining 91 events were identified as earthquakes.

To test the performance of the automatic method, the data were also analysed with the Rasmussen method, where data are manually scanned using predefined time windows and filters (Rasmussen \& Voss 2011). During this manual scanning, the z-channel was checked in two-hourly windows using a 2-9 $\mathrm{Hz}$ band pass filter, the same filter that is used to analyse the data from the seismometer at Danmarkshavn in the daily processing at GEUS. The scanning found 229 earthquakes in the study period, approximately two and a half times more earthquakes than found by the automatic method. An example of an earthquake that was not detected by the automatic method is shown in Fig. 3B. Comparison of the lists of earthquakes detected by the two methods shows that the automatic detections do not give a full subset of the manual detections, as the automatic method detected 26 events that were not found by the manual method. A review of these 26 earthquakes showed that 23 had a low signal to noise ratio in the frequency range of the band pass filter $(2-9 \mathrm{~Hz})$ used and three earthquakes were missed during the analysis.

After the detection procedure, we processed the detected events. In this process, the arrival times of the $P$ - and $S$-phases were picked and the magnitude of the signal determined. The automatic method picks $P$ - and $S$-phases and uses the duration of the signal tremor to estimate the magnitude. We reviewed the 91 earthquakes detected and found that the automatic pick of the $P$ - and $S$-phases should be adjusted for all earthquakes. Most of the adjustments were within a few seconds, but for seven of the events the automatic pick errors were several seconds. In many cases, the duration of the automatic signal located the end of the event in the last part of the $S$ wave, but in some instances the end of the duration was in the end of the $P$ wave. A similar approach was used by Agius $\&$ Galea (2011) with good results, but further improvement of the processing is required. Examples of the performance of the automatic method are shown on seismograms in Fig 3.

\section{Discussion and conclusions}

The manual detection method found two and a half times more events than the automatic detection method. Replacement of the manual method with the automatic detection of earthquakes in Greenland will therefore result in a significantly lower number of earthquake detections. The quality of earthquake risk evaluation depends on a high detection level; thus a lower number of detections will lead to a lower quality of an earthquake risk evaluation. The automatic method contributed with 26 (11\%) additional events to the manual detections, and hence a combination of the two methods may increase the number of earthquakes detected in the region. In addition, the automatic method was effective in avoiding false detections.

The automatic method gives earthquake locations and magnitude estimates, based on automatic phase picks, phase polarisation and signal duration, but the quality of this information is poorer than that obtained by manual processing. A manual review of the data will still be an important part of the quality control.

\section{Acknowledgements}

This study is part of a PhD project funded by the International Institute of Earthquake Engineering and Seismology for the first author, who was a visiting PhD student at GEUS in 2011. The Greenland Ice Sheet Monitoring Network is thanked for upgrading Station Nord.

\section{References}

Agius, M.R. \& Galea, P. 2011: A single-station automated earthquake location system at Wied Dalam Station, Malta. Seismological Research Letters 82, 545-559.

Chung, W.-Y. 2002: Earthquakes along the passive margin of Greenland: evidence for postglacial rebound control. Pure and Applied Geophysics 159, 2567-2584.

Døssing, A., Stemmerik, L., Dahl-Jensen, T. \& Schlindwein, V. 2010: Segmentation of the eastern North Greenland oblique-shear margin - regional plate tectonic implications. Earth and Planetary Science Letters 292, 239-253.

Gibbons, S. J., Ringdal, F. \& Kværna, T. 2008: Detection and characterization of seismic phases using continuous spectral estimation on incoherent and partially coherent arrays. Geophysical Journal International 172, 405-421.

Gregersen, S. 1982: Seismicity and observations of $\mathrm{Lg}$ wave attenuation in Greenland. Tectonophysics 89, 77-93.

Havskov, J. \& Alguacil, G. 2010: Instrumentation in earthquake seismology. Modern Approaches in Geophysics 22, 360 pp.

Lehmann, I. 1954: Characteristic earthquake records. Geodætisk Instituts Skrifter III. Række 18, 33 pp.

Rasmussen, H.P. \& Voss, P.H. 2011: Detection of earthquakes at the Geological Survey of Denmark and Greenland - GEUS. 42nd Nordic Seminar on Detection Seismology, Reykjavik, 5-7 October 2011. Icelandic Meteorological Office, programme and abstracts, 41 only.

Voss, P., Poulsen, S.K., Simonson, S.B. \& Gregersen, S. 2007: Seismic hazard assessment of Greenland. Geological Survey of Denmark and Greenland Bulletin 13, 57-60.

\footnotetext{
Authors' addresses

N.K. \& G.D.J., International Institute of Earthquake Engineering and Seismology, Arghavan St. 21, North Dibajee, Farmanieh Tehran $3913 / 19395$

Islamic Republic of Iran. E-mail: n.karamzadeh@iiees.ac.ir

P.H.V., Geological Survey of Denmark and Greenland, Øster Voldgade 10, DK-1350 Copenhagen K, Denmark.
} 